

University of Central Florida

STARS

$1-1-2013$

\title{
Heat transfer mechanism across few-layer graphene by molecular dynamics
}

\author{
Meng Shen \\ Patrick K. Schelling \\ University of Central Florida \\ Pawel Keblinski
}

Find similar works at: https://stars.library.ucf.edu/facultybib2010 University of Central Florida Libraries http://library.ucf.edu

This Article is brought to you for free and open access by the Faculty Bibliography at STARS. It has been accepted for inclusion in Faculty Bibliography 2010 s by an authorized administrator of STARS. For more information, please contactSTARS@ucf.edu.

\section{Recommended Citation}

Shen, Meng; Schelling, Patrick K.; and Keblinski, Pawel, "Heat transfer mechanism across few-layer graphene by molecular dynamics" (2013). Faculty Bibliography 2010s. 4687.

https://stars.library.ucf.edu/facultybib2010/4687

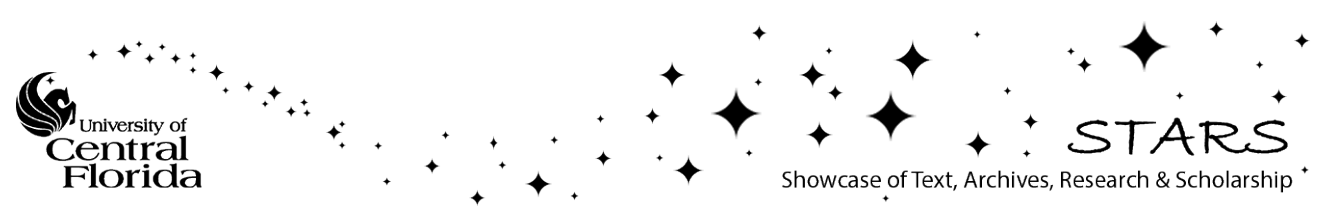




\title{
Heat transfer mechanism across few-layer graphene by molecular dynamics
}

\author{
Meng Shen, ${ }^{1}$ Patrick K. Schelling, ${ }^{2}$ and Pawel Keblinski ${ }^{1, *}$ \\ ${ }^{1}$ Department of Materials Science and Engineering and Rensselaer Nanotechnology Center, Rensselaer Polytechnic Institute, \\ Troy, New York 12180, USA \\ ${ }^{2}$ Advanced Material Processing and Analysis Center and Department of Physics, University of Central Florida, \\ 4000 Central Florida Blvd., Orlando, Florida 32816, USA
}

(Received 29 October 2012; revised manuscript received 30 May 2013; published 31 July 2013)

\begin{abstract}
We use nonequilibrium molecular dynamics to study heat transfer across structures consisting of a few layers of graphene sandwiched between silicon crystals. We find that when heat transfers from a silicon lead on one side across the graphene layers to a silicon lead on the other side, the interfacial conductance is essentially independent of the number of layers, in agreement with recent experimental findings. By contrast, wave-packet simulations show that the transmission coefficient of individual vibrational modes depends strongly on frequency and the number of graphene layers, indicating significant interference effects. This apparent contradiction is resolved by a theoretical calculation, which shows that the integrated contribution of the phonons to the interfacial thermal conductance is essentially independent of the number of layers. When one atomic layer of graphene is heated directly, the effective interfacial conductance associated with heat dissipation to the silicon substrate is much smaller. We attribute this to the resistance associated with heat transfer between high and low frequency modes within heated graphene.
\end{abstract}

DOI: 10.1103/PhysRevB.88.045444

PACS number(s): 63.22.Rc, 68.35.-p

\section{INTRODUCTION}

Heat-transfer mechanisms at the nanoscale level have been of significant fundamental interest in the recent decade. ${ }^{1}$ When feature size scales down to the phonon-mean free path, Fourier's Law is not sufficient to describe heat conduction in solids, and the solution of the Boltzmann Transport Equation (BTE) is used to elucidate thermal-transport properties. ${ }^{1-3}$ However, at further reduced sizes, such as an interface formed by a few layers of graphene, the wave nature of phonons can no longer be neglected. Methods such as BTE, which treat phonons as quasiparticles, must be replaced by more sophisticated theoretical models that capture the wave features of phonons, such as coherency and interference, ${ }^{4}$ which have been a focus of theoretical ${ }^{5-14}$ and experimental studies. ${ }^{15-20}$

Graphene has attracted a lot of attention from the research community in recent years, primarily due to its remarkable in-plane electrical and thermal conductivity. ${ }^{21,22}$ Very high in-plane thermal conductivity ${ }^{23,24}$ (up to $5300 \mathrm{~W} \mathrm{~m}^{-1} \mathrm{~K}^{-1}$ ) indicates that heat dissipation in systems involving graphene is likely limited by the out-of-plane heat transfer. While intensive studies have been carried out on the in-plane heat conduction in suspended or supported graphene, ${ }^{23-27}$ few studies have been focused on the out-of-plane heat transfer. ${ }^{28-31}$

Mao et al ${ }^{31,32}$ studied the effect of different metal substrates on the cross-plane heat transfer of graphene layers by firstprinciple method. They observed that, in general, different metal substrates result in different interfacial bonding strength and hence different interfacial thermal conductance. ${ }^{31}$ In our paper, instead of focusing on the effect of different substrates and surface functionalization, we choose $\mathrm{Si}$ as a prototype lead material, we study the heat transfer mechanism across physisorbed graphene layers with weak van der Waals interfacial bonding, ${ }^{31}$ and we investigate the role of potential interference effects.

Recent experiments on metal $/ n$-graphene/ $\mathrm{SiO}_{2}$ interfaces have shown that the interfacial heat conductance $G$ is indepen- dent of the number of graphene layers $n(1 \leqslant n \leqslant 10) .{ }^{28}$ This implies, on one hand, that phonons are ballistically transported across the graphene layers but, on the other hand, that the scattering events at metal $/ n$-graphene and $n$-graphene $/ \mathrm{SiO}_{2}$ contacts are independent, suggesting that coherent phononinterference effects are either not present or not important. By contrast, phonon interference was demonstrated in onedimensional junctions, thin films, and multilayer films both experimentally ${ }^{15,16,20,33,34}$ and theoretically. ${ }^{10,12-14,35-37}$ For example, theoretical calculations of a two-junction interface model indicated that significant phonon interference effects are expected for the case of ballistic transport between the junctions. ${ }^{36}$ With this context, it is necessary to investigate how the transmission coefficient of individual phonons affects interfacial thermal conductance.

In this work, the interfacial thermal conductance of a prototype $\mathrm{Si} / n$-graphene/Si structure was determined by a nonequilibrium molecular dynamics (NEMD) simulation. Also, transmission coefficients of individual phonons were evaluated by a wave-packet dynamics simulation and an analytical mass-spring model. Integration over all phonon frequencies and incident angles elucidated the apparent contradiction between interface thickness-independent interfacial conductance and interface thickness-dependent transmission coefficient.

A different situation arises when instead of considering heat transfer from one solid lead across graphene layers to the other solid lead, one considers heat dissipation to the solid substrate when the graphene layer is heated directly. This process is particularly relevant when an electric current in graphene generates thermal energy. ${ }^{38-45} \mathrm{Li}$ et al. theoretically studied the substrate effect on the joule heating of graphene. In his study, however, the fast equilibrium for the phonon system was assumed as a priori for simplicity. ${ }^{42}$ This type of heat transfer is discussed as a separate case later in the paper. 
The rest of the paper is organized as follows: In Sec. II, we describe the model structure in our NEMD simulation and present the results of interfacial thermal conductance of the $\mathrm{Si} / n$-graphene/Si junction. In $\mathrm{Sec}$. III, we evaluate the transmission coefficient of individual phonons by both wavepacket dynamics simulation and the analytical calculation of a mass-spring model. In Sec. IV, we show why the interfacial thermal conductance is independent of the number of layers despite the strong interference effects exhibited by individual phonons. In addition, we extend this calculation to different lead materials. In Sec. V, we investigate the effect of surface reconstruction, crystallographic orientation, and the strain of $\mathrm{Si}$ leads on heat transfer across $\mathrm{Si} / n$-graphene/Si structure. In Sec. VI, instead of studying heat transfer across graphene layers, we investigate heat dissipation from a directly heated graphene layer. Finally, in Sec. VII, we present our conclusion and provide a general discussion.

\section{MD SIMULATION OF THE INTERFACIAL CONDUCTANCE OF $\mathrm{Si} / \boldsymbol{n}$-GRAPHENE/Si INTERFACE}

We use MD simulations (LAMMPS ${ }^{46}$ package) to study heat transfer through $\mathrm{Si} / n$-graphene/Si interfaces (two-junction structure) with the number of graphene layers ranging from 1 to 10 . Crystalline $\mathrm{Si}$ is chosen as a prototype lead structure. The use of $\mathrm{Si}$ is also of practical interest because direct contact between $\mathrm{Si}$ and graphene has been used to make solar cells. ${ }^{47}$ Moreover, graphene has been grown directly on $\mathrm{Si}^{48}$ To explore the situation of the direct heating of graphene, we also study a single junction structure of graphene on a silicon substrate.

The main simulation structure was composed of one or a few graphene layers in between two crystalline silicon blocks with (l 11 1 $)$ surfaces (see Fig. 1, top panel). The cross-section dimensions were $3.06 \mathrm{~nm}$ by $5.31 \mathrm{~nm}$, and the silicon blocks are 11.2-nm long in the cross-plane $(z)$ direction. The structure details are as follows: For Si, there

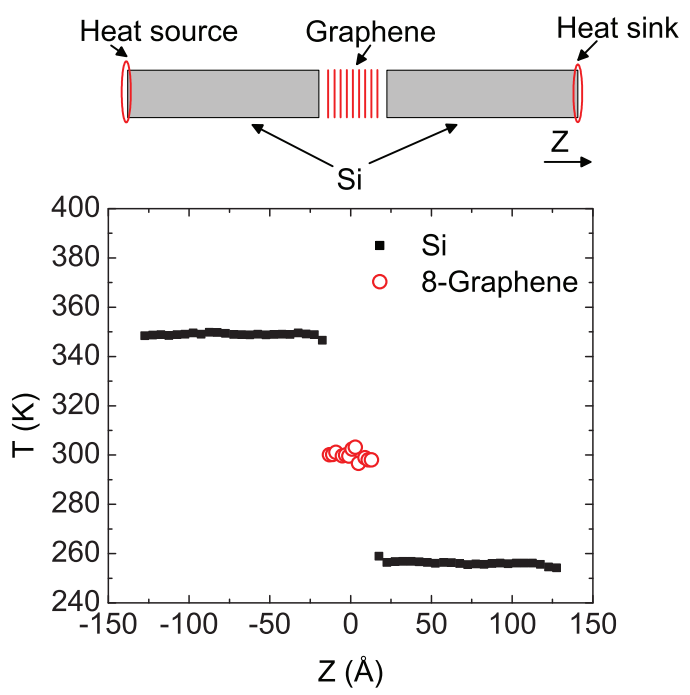

FIG. 1. (Color online) (top panel) Simulation structure and placement of heat source/sink and (bottom panel) steady state temperature profile of $\mathrm{Si} / 8$-graphene/Si structure (solid squares: $\mathrm{Si}$; open circles: graphene). are eight unit cells $(8 \times \sqrt{2} / 2$ cubic lattice constant) in the $x$ direction and eight unit cells $(8 \times \sqrt{6} / 2$ cubic lattice constant $)$ in the $y$ direction; for graphene, there are seven unit cells in the $x$ direction $(7 \times \sqrt{3}$ lattice constant), and 21 unit cells $(21 \times$ lattice constant $)$ in the $y$ direction. The cubic lattice constant of $\mathrm{Si}$ is $0.543 \mathrm{~nm}$, and a lattice constant of graphene is $0.253 \mathrm{~nm}$, corresponding to nearest-neighbor bond length of $0.146 \mathrm{~nm}$ in Tersoff graphene. The $\mathrm{Si}$ and graphene structures are made commensurate in the $x$ and $y$ directions such that there is no strain in graphene layers and a rather small strain of $0.18 \%$ in both the $x$ and $y$ directions in the Si leads. Periodic boundary conditions were applied in the $x$ and $y$ directions, and cross-sectional sizes were kept fixed at such sizes that in-plane stress is not present in the graphene layers. The free ends of the silicon blocks were exposed to vacuum, thus allowing stress relaxation in the $z$ direction.

The interatomic interactions within each silicon crystal and within each graphene layer were described by Tersoff bondorder potentials. ${ }^{49,50}$ The nonbonded interactions between $\mathrm{Si}$ atoms and $\mathrm{C}$ atoms, between $\mathrm{Si}$ atoms in different blocks, and that between $\mathrm{C}$ atoms in different layers were modeled with the 12-6 Lennard-Jones (LJ) potential, ${ }^{51} U(r)=4 \varepsilon\left((\sigma / r)^{12}-\right.$ $\left.(\sigma / r)^{6}\right)$, where $\varepsilon$ is the depth of the potential well and $\sigma$ is the distance parameter. The values of the parameters are $\varepsilon_{\mathrm{C}-\mathrm{C}}=$ $0.003 \mathrm{eV}, \sigma_{\mathrm{C}-\mathrm{C}}=3.41 \AA, \varepsilon_{\mathrm{Si}-\mathrm{Si}}=0.01182 \mathrm{eV}, \sigma_{\mathrm{Si}-\mathrm{Si}}=3.7 \AA$, $\varepsilon_{\mathrm{C}-\mathrm{Si}}=0.005955 \mathrm{eV}$, and $\sigma_{\mathrm{C}-\mathrm{Si}}=3.555 \AA,{ }^{52,53}$ where we calculated Si-C interaction parameters using Lorentz-Berthelot mixing rules. ${ }^{54}$ The LJ potential has been used to describe van der Waals forces between substrate and graphene in the case of physisorbed interfaces, ${ }^{25,30,55,56}$ which applies to the case of the transferred graphene/Si interface,${ }^{47}$ and has been demonstrated to describe thermal coupling between graphene layers and between graphene and the substrate well in comparison with experiments. $^{25,57,58}$ Also using the same potential in MD simulations, the predicted thermal conductivity of multigraphene thin films ${ }^{59}$ is in good agreement with experimental results. ${ }^{60}$

We used the so-called direct method ${ }^{61}$ to study thermal transport across the interface. The heat source/sink was applied to the free ends of the $\mathrm{Si}$ blocks, as illustrated in Fig. 1 (top panel). A constant heat power $P=38.4 \mathrm{nW}$ was added to/removed from the heat source/sink by scaling velocities of the atoms in the heat source/sink regions at every time step. A steady state was established after $\sim 2.5 \mathrm{~ns}$, and the temperature profiles, as shown in Fig. 1 (bottom panel), were obtained by time averaging over $\sim 0.5 \mathrm{~ns}\left(\sim 10^{6} \mathrm{MD}\right.$ steps $)$.

No obvious temperature gradient is present within graphene layers (Fig. 1, bottom panel), indicating ballistic phonon transport. From the temperature drop at the interface, $\Delta T$ (Fig. 1, bottom panel), we calculated the interfacial thermal conductance, $G$, as, $G=P /(A \Delta T)$, with $A$ being the crosssectional area. In selected simulations, we doubled the length of the Si blocks and, within the statistical error, observed the same values of the interfacial conductance, indicating that finite-size effects are negligible.

The interfacial thermal conductance for the $\mathrm{Si} / n$ graphene/Si structure as a function of the number of graphene layers is presented in Fig. 2 (solid squares). The double junction has a conductance of about $20 \mathrm{MW} \mathrm{m}^{-2} \mathrm{~K}^{-1}$ and is essentially independent of the number of graphene layers. 




FIG. 2. (Color online) Thermal conductance of the $\mathrm{Si}(111) / n$ graphene/Si (111) structure (solid squares) and $\mathrm{Si}(100) / n$ graphene/Si (100) structure (solid circles) in the cross-plane direction as a function of number of graphene layers from NEMD.

This fact is consistent with experimental observations ${ }^{28}$ and suggests that phonons travel ballistically across the graphene layers. There are, however, no obvious signs of coherent phonon interference, such as nonmonotonic conductance dependence on the number of graphene layers.

Ballistic phonon transport is consistent with the temperature profile in Fig. 1, showing that the majority of the temperature drop is at the Si-graphene interface, while there is almost a flat profile for graphene. In fact, using the same model for graphene, Wei et al. determined cross-plane thermal conductivity of nanometer-thick graphite thin films to be in the $0.1-1.5 \mathrm{~W} \mathrm{~m}^{-1} \mathrm{~K}^{-1}$ range (see Ref. 59), which is consistent with the recently reported experimental value of $0.7 \mathrm{~W} \mathrm{~m}^{-1}$ $\mathrm{K}^{-1}$ for 35 -nm-thick graphite thin film. ${ }^{60}$ These conductivity values are much smaller than that characterizing bulk graphite, which is about $7.0 \mathrm{~W} \mathrm{~m}^{-1} \mathrm{~K}^{-1}$ in the cross-plane direction, ${ }^{62}$ indicating that the phonon-mean free path in the cross-plane direction is quite large.

This can be further corroborated by an estimate of the crossplane phonon-mean free path. From kinetic theory, ${ }^{63}$ thermal conductivity is expressed as

$$
k=\frac{1}{3} C v l,
$$

where $C$ is specific heat, $v$ is phonon velocity, and $l$ is the phonon-mean free path. Equation (1) is based on an isotropic assumption. However, graphite is highly anisotropic in the group velocities of longitudinal acoustic (LA) and transverse acoustic (TA) modes ${ }^{64,65}$; therefore, we modify Eq. (1) to account for LA and TA modes explicitly:

$$
k=\frac{1}{3}\left(C_{l} v_{l}+2 C_{t} v_{t}\right) l,
$$

where $C_{l}$ and $C_{t}$ are heat capacities distributed in LA and TA modes, respectively, and $v_{l}$ and $v_{t}$ are group velocities for LA and TA modes, respectively. The factor of two comes from two TA modes per $k$-point. The specific heat of graphite is $1.42 \times$ $10^{6} \mathrm{~J} \mathrm{~K}^{-1} \mathrm{~m}^{-3}$ (see Refs. 60 and 66), and the cross-plane thermal conductivity of single-crystal bulk graphite is about $7 \mathrm{~W} \mathrm{~m}^{-1} \mathrm{~K}^{-1}$ (see Refs. 62 and 67) The group velocities of cross-plane LA phonons is $4140 \mathrm{~m} \mathrm{~s}^{-1}$ from inelastic x-ray scattering measurements, ${ }^{68}$ and that of TA phonons is $1257 \mathrm{~m} \mathrm{~s}^{-1}$ from inelastic neutron-scattering measurements. ${ }^{64}$ Based on the above parameters and considering every LA mode is accompanied by two TA modes, we estimate the mean free path of phonons in graphite in the cross-plane direction to be $\sim 6.7 \mathrm{~nm}$, which is much longer than the thickness of multilayer graphene films $(\leqslant 3.335 \mathrm{~nm})$ used in our studies.

In addition, the total heat capacity used in Eq. (2) was measured at room temperature. ${ }^{66}$ Due to weak van der Waals bonding between graphene layers, however, only low frequency phonons $\left(\leqslant 4 \mathrm{THz},{ }^{64}\right.$ corresponding to the Debye temperature of $180.5 \mathrm{~K})^{69}$ couple well across the interface ${ }^{70}$ and make considerable contributions to cross-plane thermal conductivity in graphite ${ }^{71}$ while the majority of vibrational modes in graphite have high frequencies due to rigid $\mathrm{C}$ - $\mathrm{C}$ bonding and small atomic mass. Therefore, it warrants replacing the heat capacity measured at room temperature ${ }^{66}$ used in Eq. (2) by a heat capacity contributed by low frequency phonons. In fact, graphite specific heat measured at $200 \mathrm{~K}$ (slightly larger than $180.5 \mathrm{~K}$, graphite cross-plane Debye temperature ${ }^{69}$ ) is $9.13 \times 10^{5} \mathrm{~J} \mathrm{~K}^{-1} \mathrm{~m}^{-3}$ (see Ref. 72). Substituting this value into Eq. (2), the mean free path is estimated to be $10.4 \mathrm{~nm}$.

\section{TRANSMISSION COEFFICIENT OF INDIVIDUAL PHONONS: WAVE-PACKET DYNAMICS SIMULATION AND A MASS-SPRING MODEL}

To understand the lack of any signature of phonon interference in the interfacial conductance, we performed wave-packet MD simulations..$^{73}$ In these simulations, a phonon wave packet with a well-defined polarization centered at a narrow frequency range was introduced into one silicon lead and launched toward the graphene interface. After the completion of the interfacial scattering process, we evaluated the transmission coefficients defined as the ratio of the transmitted energy to the incident phonon energy.

In Fig. 3, we show snapshots of displacement of propagating LA wave packets at different times for wave packets centered at $2.67 \mathrm{THz}$ with a corresponding wavelength of $3.36 \mathrm{~nm}$ in Si leads ${ }^{74}$ [Fig. 3(a)] and at $2.86 \mathrm{THz}$ with a corresponding wavelength of $3.14 \mathrm{~nm}$ in Si leads [Fig. 3(b)]. Only a small portion of the $2.67 \mathrm{THz}$ wave-packet energy is transmitted [Fig. 3(a), bottom panel], corresponding to a transmission coefficient of 0.03 associated with constructive interference of the reflected phonons; on the other hand, almost half of the $2.86 \mathrm{THz}$ wave-packet energy is transmitted to the $\mathrm{Si}$ lead on the other side [Fig. 3(b), bottom panel], with a high transmission coefficient of 0.42 associated with destructive interference of the reflected phonons.

This strong frequency dependence of the phonon transmission indicates a coherent phonon-interference effect. This is further illustrated by the plot of the transmission coefficient as a function of frequency, shown in Fig. 4(a). In particular, in Fig. 4(a) we show the transmission coefficient, $\alpha$, for LA phonons involving junctions with one, five, and eight graphene layers. In all cases, $\alpha$ exhibits strongly nonmonotonic behavior as a function of frequency with well-defined maxima and a decaying envelope. The presence of the peaks can be understood in terms of phonon-interference effects. Analogous to the transmission of mechanical and optical waves, when 




(a)

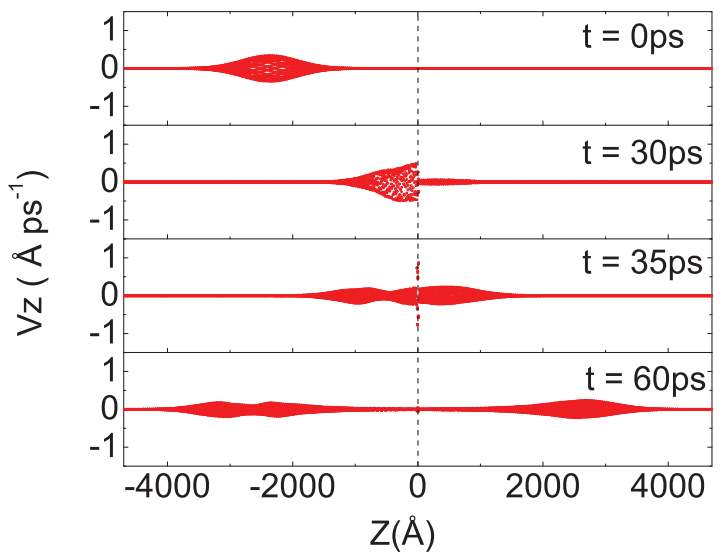

(b)

FIG. 3. (Color online) Snapshots of wave packets launched in the left Si-lead centered at (a) $2.67 \mathrm{THz}$ (wavelength $=3.36 \mathrm{~nm}$ in $\mathrm{Si}$ ) and (b) $2.86 \mathrm{THz}$ (wavelength $=3.14 \mathrm{~nm}$ in $\mathrm{Si}$ ) through $\mathrm{Si} / 8$ graphene/Si structure at different times. Dashed lines denote location of the interface.

the two wave packets reflected from $\mathrm{Si} / \mathrm{C}$ and $\mathrm{C} / \mathrm{Si}$ contacts are out of phase, reflection is minimized, ${ }^{75}$ and transmission is maximized. Therefore, the peaks in Fig. 4 correspond to destructive interference for the reflected wave, while valleys correspond to constructive interference for reflected wave packets.

To emphasize the generality of the behavior shown in Fig. 4(a), we evaluated $\alpha$ as a function of frequency by analytical calculations of a simple one-dimensional model of masses connected by springs. The model includes a center region (mimicking graphene layers) sandwiched between two semi-infinite leads (mimicking silicon). In this model, masses are connected by harmonic springs, where $m_{1}=m_{3}, m_{2}$ and $k_{1}=k_{3}, k_{2}$ are masses and spring constants in the left, right, and center part, respectively; $k_{12}=k_{23}$ are spring constants with which the semi-infinite chains are coupled with the center part. ${ }^{35}$ We parameterized the $k_{1}$ and $k_{2}$ spring constants and masses to reproduce the speed of sound of $\mathrm{Si}$ and graphite in the cross-plane direction. The value of the interfacial spring constant, $k_{12}$, was selected to model the Si-C LJ potential bonding strength used in the MD simulations.

The transmission coefficients for junction models mimicking one, three, five, and eight graphene-layer structures

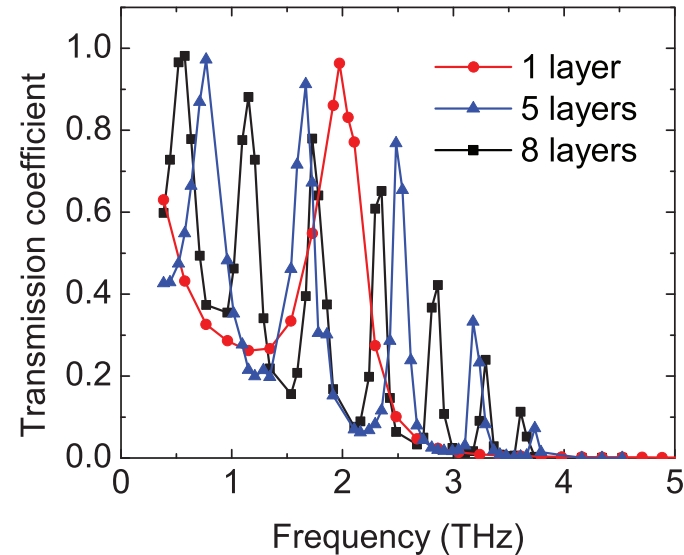

(a)

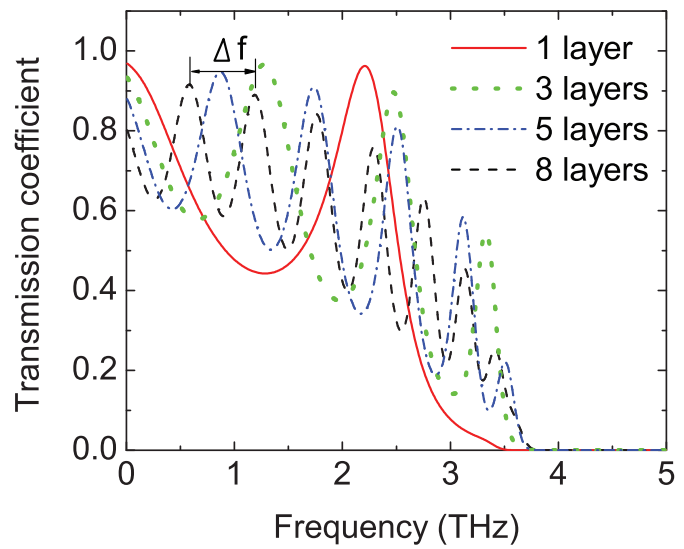

(b)

FIG. 4. (Color online) Transmission coefficient as a function of frequency obtained from (a) wave-packet dynamics simulations for one graphene layer (solid circles), five graphene layers (solid triangles), and eight graphene layers (solid squares); and (b) analytical calculation considering a Gaussian wave packet in a one-dimensional mass-spring model with a double junction for one (solid line), three (dotted line), five (dashed dot line), and eight (dashed line) graphene layers. $f$ denotes oscillation period in frequency.

calculated by the scattering boundary method ${ }^{35}$ are shown in Fig. 4(b). To directly compare the results with the MD simulations in Fig. 4(b) at each frequency, we show the average transmission coefficients over phonons representing a wave packet used in the MD simulations. The overall behavior of $\alpha$ as a function of frequency is strikingly similar to that obtained by wave-packet MD simulations [Fig. 4(a)].

In particular, the oscillatory period, $\Delta f$, in the frequency dependence of the transmission coefficient decreases with an increasing number of layers [see Fig. 4(a) and Fig. 4(b)]. This can be understood in the following: The time corresponding to $\Delta f$ is given by $1 / \Delta f$, and the associated wave-propagation distance is $v / \Delta f$, where $v$ is the speed of sound (in graphene). In Fig. 5, we plot this propagation distance as a function of graphene-layer thickness and observe a linear relationship with a slope equal to about two. The fact that the propagation distance is twice the film thickness can be interpreted as the propagation of the phonon wave back and forth across the graphene layer. The behavior is exactly analogous to that 


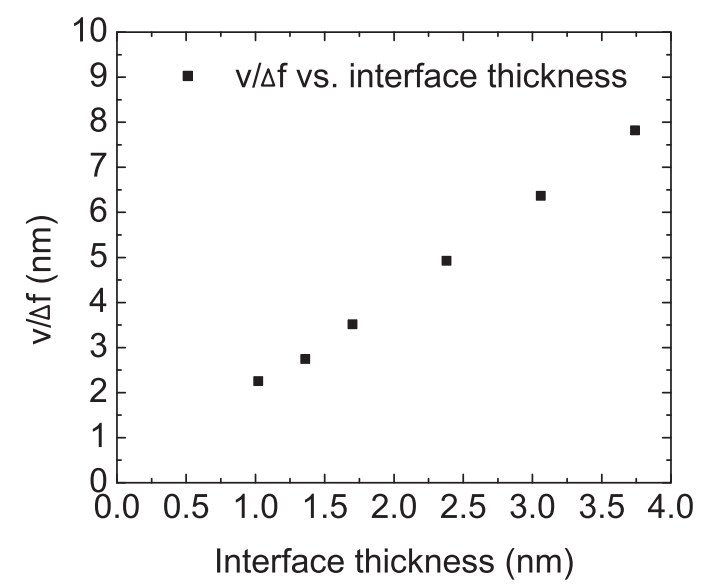

FIG. 5. Speed of sound divided by the period in frequency oscillations of transmission coefficient, $v / f$, as a function of graphene-layer thickness. The linear dependence is characterized by a slope of 2.08.

characterizing optical transmission and reflection from a thin coating.

\section{CALCULATION OF INTERFACIAL THERMAL CONDUCTANCE BY INTEGRATING OVER INDIVIDUAL PHONONS}

Both wave-packet MD simulations and analytical calculations show strongly oscillatory dependence of $\alpha$ on a frequency indicative of significant interference effects. On the contrary, the interfacial conductance obtained by MD simulations (Fig. 2) is independent of the number of graphene layers, suggesting lack of interference. To clarify this apparent contradiction, we estimate the interfacial thermal conductance via an integral formula and use the Debye approximation for phonon dispersion ${ }^{76}$ :

$$
\begin{aligned}
G(T)= & \int_{0}^{\omega_{D}} d \omega \int_{0}^{\frac{\pi}{2}} d \theta \sin (\theta) c \cos (\theta) \alpha(\omega \cos (\theta)) \hbar \omega \\
& \times \frac{\omega^{2}}{2 \pi^{2} v^{3}} \frac{d N(\omega, T)}{d T},
\end{aligned}
$$

where $G(T)$ is interfacial thermal conductance, and the integration is over an angular frequency, $\omega$, (from zero to the Debye frequency) and over the incident angle $\theta$. The phonon velocity is $v$, and $\hbar \omega$ is the phonon energy, where $\hbar=\frac{h}{2 \pi}$ and $h$ is Plank's constant. $\frac{\omega^{2}}{2 \pi^{2} v^{3}}$ is the Debye phonon density of states, $\omega_{D}$ is the Debye frequency, and $N(\omega, T)$ is phonon-occupation factor. To compare with classical dynamics MD simulation results, we use the Boltzmann statistics occupation number $N(\omega, T)=k_{B} T /(\hbar \omega)$, where $k_{B}$ is the Boltzmann constant. In evaluating the above integral, we assume that the transmission coefficient $\alpha(\omega \cos (\theta))$ depends only on the component of the wave vector normal to the interface.

The interfacial conductance normalized by the value in the case where transmission coefficient is unity for all modes (i.e., the radiation limit $^{76}$ ) is almost constant for different numbers of graphene layers ranging from $n=1$ to $n=10$, as shown in Fig. 6. More detailed analysis indicates that this essentially constant value originates from averaging out of the oscillatory features in the transmission coefficient by integrating over

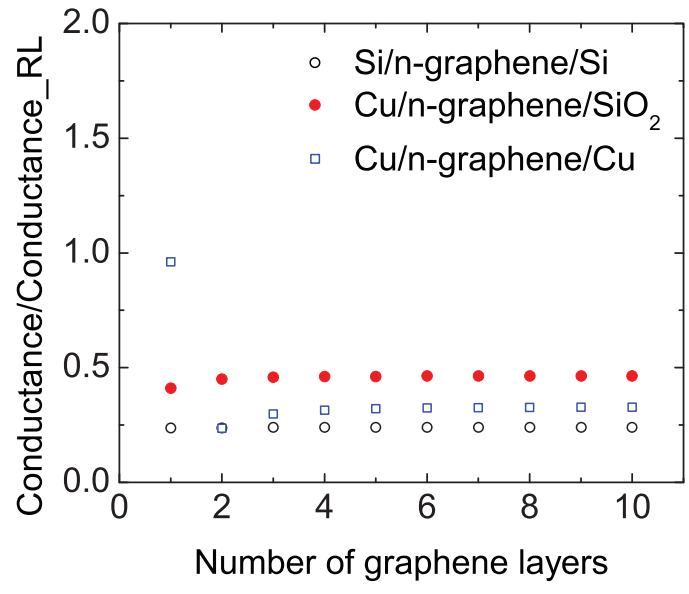

FIG. 6. (Color online) Interfacial thermal conductance normalized by the radiation limit value as a function of number of graphene layers calculated via the integration over all the phonons given by Eq. (2) for $\mathrm{Si} / n$-graphene/Si (open circles), $\mathrm{Cu} / n$ graphene/ $/ \mathrm{SiO}_{2}$ (solid circles), and $\mathrm{Cu} / n$-graphene/Cu (open squares) interfaces.

frequencies with further averaging over incident angles. This result resolves the apparent contradiction. Namely, while the transmission of phonons depends strongly on frequency and the number of graphene layers, integration over all frequencies and incident angles results in an interfacial conductance nearly independent of the number of graphene layers.

Since we established that phonon transmission coefficients obtained via MD simulations match with those evaluated analytically from a simple mass and spring model very well, we can easily analyze the interfacial thermal conductance across graphene involving different lead materials. In particular, a $\mathrm{Cu} / n$-graphene $/ \mathrm{SiO}_{2}$ interfacial thermal conductance evaluated as such exhibits a slightly lower conductance for $N=1$ and $N$ independent conductance for $N>1$, consistent with experimental measurement ${ }^{28}$ (see Fig. 6). It is worth noting that the value of the interfacial spring constants, $k_{12}$ and $k_{23}$ are selected to model $\mathrm{Cu}-\mathrm{C}^{30,55}$ and $\mathrm{C}-\mathrm{SiO}_{2}{ }^{77}$ bonding strength, respectively.

Interestingly, the conductance of $\mathrm{Cu} / \mathrm{n}$-graphene/Cu interface is independent of the number of graphene layers, $N$ but only for $N>2$. For a single atomic layer of graphene, the conductance is four times larger than for thicker layers (see Fig. 6). This is qualitatively consistent with Chang's observation ${ }^{30}$ that interfacial conductance decreases from value of $N=1$ to $N=3$ and remains constant afterwards up to $N=10$. We attribute the large conductance of a single-layer structure with $\mathrm{Cu}$ leads to strong interfacial interactions between $\mathrm{Cu}$ and $\mathrm{C}$, which is comparable with the strength of the $\mathrm{Cu}-\mathrm{Cu}$ interactions. Consequently, in the case of $N=1$, phonon transmission is close to unity across the whole frequency range. However, for $N>1$, weak interaction between graphene layers leads to discontinuity in the bonding strength and lowers the interfacial thermal conductance.

In the case of interfaces involving a copper lead, the effect of the electrons on the interfacial conductance needs to be addressed. In Majumdar and Reddy's ${ }^{78}$ model, it is assumed that phonons in insulators couple with phonons in the metal, 
and then electron-phonon coupling in the metal transfers the heat to electrons. Consequently, the thermal resistance associated with electron-phonon coupling is connected in series with the resistance due to phonon-phonon coupling. Majumdar and Reddy $^{78}$ estimated that the conductance associated with the electron-phonon coupling is of the order of $\mathrm{GW} \mathrm{m}{ }^{-2} \mathrm{~K}^{-1}$; thus, the associated resistance is insignificant as compared with phonon-associated resistance at the graphene/substrate interface. $^{31,79,80}$ The phonon-phonon interfacial conductance thus provides a good estimate of the overall interfacial conductance.

\section{EFFECT OF SURFACE RECONSTRUCTION, CRYSTALLOGRAPHIC ORIENTATION, AND STRAIN OF SI LEADS}

To inquire into the generality of our findings reported so far, we evaluated the potential effects of Si surface reconstruction on the interfacial thermal conductance. In fact, the Tersoff potential used in this study was employed to investigate surface reconstruction of $\mathrm{Si}^{81,82}$ Accordingly, we prepared $\mathrm{Si} / n$-graphene/Si samples with (111) reconstructed surfaces.

As shown in Fig. 7(a), the phonon transmission coefficients for the reconstructed surface overlap with the data for unreconstructed surfaces almost perfectly, indicating that surface reconstruction does not play an important role in heat flow across $\mathrm{Si} / n-\mathrm{Gr} / \mathrm{Si}$ interface with weak van der Waals interfacial interaction. ${ }^{47}$ We note that in the case of covalently bonded interfaces, reconstruction could play an important role in altering interfacial bonding and structure. ${ }^{83}$

In order to investigate a possible role of the crystallography of the Si surface, in addition to (111) surfaces, we computed the interfacial conductance for selected structures with (100)Si/n-graphene/(100)Si interfaces. Similar to the (111) Si case, the interfacial thermal conductance is more or less independent of the number of graphene layers. There is only a small quantitative difference $(<10 \%)$ between the $(111)$ $\mathrm{Si}$ and slightly larger (100) $\mathrm{Si}$-associated interfacial thermal conductance (see Fig. 2). We also consistently observed that the transmission coefficients for (100) Si leads [solid triangles in Fig. 7(a) and dashed curves in Fig. 7(b)] are slightly larger than for (111) Si leads [solid squares in Fig. 7(a) and solid lines in Fig. 7(b)]. These results are also consistent with recent experimental and theoretical studies that claimed that interfacial thermal resistance does not show a strong dependency on crystallographic orientation of cubic lattices. ${ }^{84,85}$

To employ periodic boundary conditions in the in-plane direction, we slightly strained $\mathrm{Si}$ leads as to have unstrained graphene. This raises a concern of the possible effect of strain on the interfacial thermal conductance. To address this concern, we also performed NEMD on a structure with $2 \%$ in-plane strain in $\mathrm{Si}$ (as opposed to $0.18 \%$ strain in the original structure). The observed difference in the interfacial thermal conductance for the two structures was essentially within the statistical error. This limited effect of the lateral strain is likely also associated with weak van der Waals bonding. In the case of covalently bonded interfaces, strain could play an important role in altering interfacial bonding and structure. ${ }^{83}$



(a)



(b)

FIG. 7. (Color online) (a) Comparison between transmission coefficients of structures with Si (111) leads without surface reconstruction (solid squares), with surface reconstruction (solid circles), and of structures with Si (100) leads (solid triangles) for interfaces with one graphene layer (top panel) and with eight graphene layers (bottom panel) from wave-packet dynamics simulation results. (b) Transmission coefficient of structures with Si (111) leads (solid lines) and with Si (100) leads (dashed lines) for interfaces with one graphene layer (top panel) and eight graphene layers (bottom panel) from mass-spring model calculations.

\section{MD SIMULATION OF HEAT TRANSFER BETWEEN DIRECTLY HEATED GRAPHENE AND THE SI SUBSTRATE}

Finally, to mimic the situation when direct heating of the graphene layers occurs due to Joule heating resulting from an electrical current in the graphene, we performed MD simulations in which the heat source was placed in one-layer graphene film and the heat sink in the end of the silicon substrate. In this setup, we obtained an interfacial conductance of about $8 \mathrm{MW} \mathrm{m} \mathrm{m}^{-2} \mathrm{~K}^{-1}$, i.e., several times smaller than that obtained in the case where heat flows across the graphene layer. The same phenomenon has been observed by L. Hu et al. ${ }^{29}$ in the case of the heat transfer between graphene and a polymer substrate.

This effect can be understood by the following consideration: ${ }^{29}$ By directly rescaling atomic velocities in 


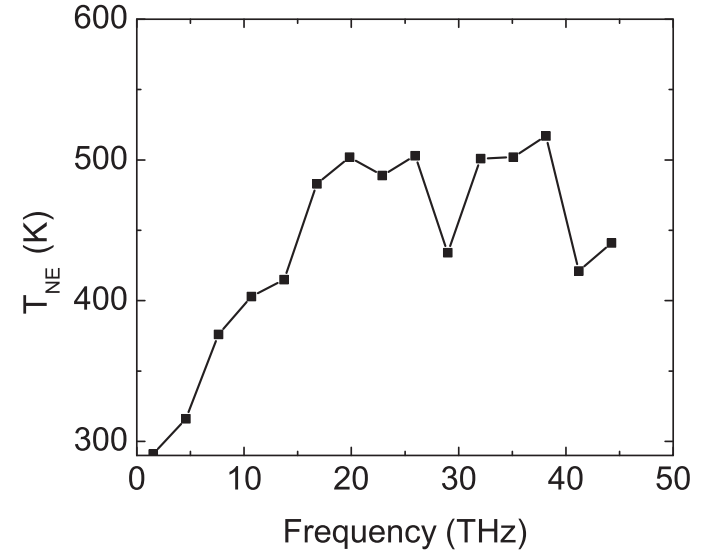

FIG. 8. Spectral temperature of phonons in a single-graphene layer as a function of frequency for steady-state simulation when the graphene layer is directly heated by velocity rescaling. Silicon temperature in this simulation is $280 \mathrm{~K}$.

graphene, energy is more or less uniformly injected into all available modes. However, the thermal energy transfer at weakly bonded interfaces associated with graphene is dominated by modes with relatively low frequencies $(\leqslant 4$ $\mathrm{THz})^{29}$, In graphene layers, due to strong C-C covalent bonding and small mass of the $\mathrm{C}$ atoms, the vast majority of the modes have much higher frequencies. ${ }^{29,67,68}$ Therefore, by atomic velocity rescaling, most of the energy is pumped to modes that do not directly participate in the heat transfer across the interface. ${ }^{29}$ As a result, heat injected to high frequency phonons has to first decay to low frequency phonons within graphene and then pass energy across the soft interface through low frequency phonons. ${ }^{29,86}$

To further support this claim, we show an average mode temperature ${ }^{29,87}$ as a function of frequency in a directly heated graphene layer in Fig. 8. According to Fig. 8, high frequency modes have much higher temperature than low frequency modes, which are more or less in thermal equilibrium with the Si substrate.

Consequently, in the case of the directly heated graphene, we can consider the effective thermal conductance as arising from two resistances in series: an internal resistance associated with high to low frequency heat transfer within the graphene and an external resistance associated with heat flow out of the graphene to the outside material, mostly via low frequency modes. ${ }^{29}$

\section{SUMMARY AND CONCLUSIONS}

In conclusion, we elucidated the heat conduction mechanism through a few graphene layers with a combination of NEMD, wave-packet dynamics simulation, and theoretical calculation. For individual phonons, we have shown clear evidence of the interference effects of coherent phonons that depend on both frequency and the number of graphene layers. This suggested that the interfacial conductance might depend on the number of graphene layers. However, the interfacial conductance was found to be essentially independent of the number of graphene layers. This contradiction was resolved by computing the interfacial conductance via integration over all phonons over frequency and angles. In particular, although coherent interference is present for individual modes, the overall effect is averaged out during integration, and the computed conductance does not depend on the number of graphene layers. This behavior is quite general for various combinations of solid leads sandwiching graphene layers. In some special cases, such as a $\mathrm{Cu}$-graphene- $\mathrm{Cu}$ interface, a good match in the bulk and interfacial force constants in the case of a single atomic layer of graphene $(N=1)$ leads to larger thermal conductance. However, for $N>2$, even in this case the interfacial thermal conductance becomes $N$ independent.

When a graphene layer is heated directly, the contact thermal conductance of graphene/Si is five times smaller than when heat is transported from one Si lead to the Si lead on the other side of graphene layers. The small conductance for the former is attributed to internal resistance between high frequency phonons and low frequency phonons within a graphene layer, with the former possibly excited by electric current.

\section{ACKNOWLEDGMENTS}

This work was supported by the US Air Force Office of Scientific Research Grant No. MURI FA9550-08-1-0407. P.K. was supported by USA Research Center "Alliance for MultiScale Modeling of Electronic Materials for an Energy Efficient Army". We thank Dr. E. Pop from University of Illinois at Urbana, Champaign, for discussions. We acknowledge Dr. L. F. Zhang from the National University of Singapore for providing the code of $1 \mathrm{D}$ chain model.
*Corresponding author: keblip@ @rpi.edu

${ }^{1}$ D. G. Cahill, W. K. Ford, K. E. Goodson, G. D. Mahan, A. Majundar, H. J. Maris, R. Merlin, and S. R. Phillpot, J. Appl. Phys. 93, 793 (2003).

${ }^{2}$ J. M. Ziman, Electrons and Phonons (Clarendon, Oxford, 1960).

${ }^{3}$ A. Majumdar, J. Heat Transfer 115, 7 (1993).

${ }^{4}$ S. Volz and P. O. Chapuis, J. Appl. Phys. 103, 034306 (2006).

${ }^{5}$ B. Yang and G. Chen, Phys. Rev. B 67, 195311 (2003).

${ }^{6}$ S. K. Saha and L. Shi, J. Appl. Phys. 101, 074304 (2007).

${ }^{7}$ L. Wang and B. W. Li, Phys. Rev. Lett. 101, 267203 (2008).
${ }^{8}$ E. S. Landry, M. I. Hussein, and A. J. H. McGaughey, Phys. Rev. B 77, 184302 (2008).

${ }^{9}$ L. Sun and J. Y. Murthy, J. Heat Transfer 132, 102403 (2010).

${ }^{10} \mathrm{G}$. Chen, J. Heat Transfer 121, 945 (1999).

${ }^{11} \mathrm{~S}$. Amoudache, R. Tigrine, A. Khater, and B. Bourahla, Eur. Phys. J. B 73, 405 (2010).

${ }^{12}$ P. E. Hopkins and J. R. Serrano, Phys. Rev. B 80, 201408 (2009).

${ }^{13}$ C. H. Baker, D. A. Jordan, and P. M. Norris, Phys. Rev. B 86, 104306 (2012)

${ }^{14}$ Y. H. Yan and H. Zhao, J. Appl. Phys. 111, 113531 (2012).

${ }^{15}$ C. H. Anderson and E. S. Sabisky, Phys. Rev. Lett. 24, 1049 (1970). 
${ }^{16}$ B. L. Blackford, Phys. Rev. Lett. 28, 414 (1972).

${ }^{17}$ M. N. Luckyanova, J. Garg, K. Esfarjani, A. Jandl, M. T. Bulsara, A. J. Schmidt, A. J. Minnich, S. Chen, M. S. Dresselhaus, Z. F. Ren, E. A. Fitzgerald, and G. Chen, Science 338, 936 (2012).

${ }^{18}$ C. Colvard, R. Merlin, M. V. Klein, and A. C. Gossard, Phys. Rev. Lett. 45, 298 (1980).

${ }^{19}$ J. Wang, Y. Hashimoto, J. Kono, A. Oiwa, H. Munekata, G. D. Sanders, and C. J. Stanton, Phys. Rev. B 72, 153311 (2005).

${ }^{20}$ V. Narayanamurti, H. L. Störmer, M. A. Chin, A. C. Gossard, and W. Wiegmann, Phys. Rev. Lett. 43, 2012 (1979).

${ }^{21}$ A. K. Geim and K. S. Novoselov, Nat. Mater. 6, 183 (2007).

${ }^{22}$ A. K. Geim, Science 324, 1530 (2009).

${ }^{23}$ A. A. Balandin, S. Ghosh, W. Z. Bao, I. Calizo, D. Teweldebrhan, F. Miao, and C. N. Lau, Nano Lett. 8, 902 (2008).

${ }^{24}$ J. H. Seol, I. Jo, A. L. Moore, L. Lindsay, Z. H. Aitken, M. T. Pettes, X. S. Li, Z. Yao, R. Huang, D. Broido, N. Mingo, R. S. Ruoff, and L. Shi, Science 328, 213 (2010).

${ }^{25}$ Z. Y. Ong and E. Pop, Phys. Rev. B 84, 075471 (2011).

${ }^{26} \mathrm{R}$. Prasher, Science 9, 185 (2010).

${ }^{27}$ L. Lindsay, D. A. Broido, and N. Mingo, Phys. Rev. B 82, 115427 (2010).

${ }^{28}$ Y. K. Koh, M. H. Bae, D. G. Cahill, and E. Pop, Nano Lett. 10, 4363 (2010)

${ }^{29}$ L. Hu, T. Desai, and P. Keblinski, Phys. Rev. B 83, 195423 (2011).

${ }^{30}$ S. W. Chang, A. K. Nair, and M. J. Buehler, J. Phys.: Condens. Matter 24, 245301 (2012).

${ }^{31}$ R. Mao, B. D. Kong, C. Gong, S. Xu, J. Jayasekera, K. Cho, and K. W. Kim, Phys. Rev. B 87, 165410 (2013).

${ }^{32}$ R. Mao, B. D. Kong, K. W. Kim, T. Jayasekera, A. Calzolari, and M. Nardelli, Appl. Phys. Lett. 101, 113111 (2012).

${ }^{33}$ I. Savic, N. Mingo, and D. A. Stewart, Phys. Rev. Lett. 101, 165502 (2008).

${ }^{34}$ M. V. Simkin and G. D. Mahan, Phys. Rev. Lett. 84, 927 (2000).

${ }^{35}$ L. F. Zhang, P. Keblinski, J. S. Wang, and B. W. Li, Phys. Rev. B 83, 064303 (2011).

${ }^{36}$ L. Hu, L. Zhang, M. Hu, J. S. Wang, B. W. Li, and P. Keblinski, Phys. Rev. B 81, 235427 (2010).

${ }^{37}$ Z. T. Tian, B. E. White, and Y. Sun, Appl. Phys. Lett. 96, 263113 (2010).

${ }^{38}$ E. H. Hwang and S. Das Sarma, Phys. Rev. B 77, 115449 (2008).

${ }^{39}$ E. Tiras, S. Ardali, T. Tiras, E. Arslan, S. Cakmakyapan, O. Kazar, J. Hanssan, E. Janzén, and E. Ozbay, J. Appl. Phys. 113, 043708 (2013).

${ }^{40}$ R. Bistritzer and A. H. MacDonald, Phys. Rev. Lett. 102, 206410 (2009).

${ }^{41}$ A. C. Betz, F. Vialla, D. Brunel, C. Voisin, M. Picher, A. Cavanna, A. Madouri, G. Fève, J.-M. Berroir, B. Plaçais, and E. Pallecchi, Phys. Rev. Lett. 109, 056805 (2012).

${ }^{42}$ X. Li, B. D. Kong, J. M. Zavada, and K. W. Kim, Appl. Phys. Lett. 99, 233114 (2011).

${ }^{43}$ X. Li, E. A. Barry, J. M. Zavada, M. Nardelli, and K. W. Kim, Appl. Phys. Lett. 97, 232105 (2010).

${ }^{44}$ A. Barreiro, M. Lazzeri, J. Moser, F. Mauri, and A. Bachtold, Phys. Rev. Lett. 103, 076601 (2009).

${ }^{45}$ T. Fang, A. Konar, H. L. Xing, and D. Jena, Phys. Rev. B 84, 125450 (2011).

${ }^{46}$ S. Plimpton, J. Comput. Phys. 117, 1 (1995).

${ }^{47}$ X. M. Li, H. W. Zhu, K. L. Wang, A. Y. Cao, J. Q. Wei, C. Y. Li, Y. Jia, Z. Li, X. Li, and D. H. Wu, Adv. Mater. 22, 2743 (2010).
${ }^{48}$ D. Wei and X. Xu, Appl. Phys. Lett. 100, 023110 (2012).

${ }^{49}$ J. Tersoff, Phys Rev B 37, 6991 (1988).

${ }^{50}$ J. Tersoff, Phys Rev B 39, 5566 (1989); 41, 3248(E) (1990).

${ }^{51}$ W. L. Jorgensen, J. Chandrasekhar, J. D. Madura, R. W. Impey, and M. L. Klein, J. Chem. Phys. 79, 926 (1983).

${ }^{52}$ W. J. Evans and P. Keblinski, Nanotechnology 21, 475704 (2010).

${ }^{53}$ A. E. Galashev, V. A. Polukhin, I. A. Izmodenov, and O. R. Rakhmanova, Glass Phys. Chem. 33, 86 (2007).

${ }^{54}$ M. P. Allen and D. J. Tildesley, Computer Simulation of Liquids (Oxford Science Publications, New York, 1986).

${ }^{55}$ Y. F. Guo and W. L. Guo, Nanotechnology 17, 4726 (2006).

${ }^{56}$ S. P. Huang, D. S. Mainardi, and P. B. Balbuena, Surf. Sci. 545, 163 (2003).

${ }^{57}$ L. Lindsay, D. A. Broido, and N. Mingo, Phys. Rev. B 83, 235428 (2011).

${ }^{58}$ D. L. Nika, S. Ghosh, E. P. Pokatilov, and A. A. Balandin, Appl. Phys. Lett. 94, 203103 (2009).

${ }^{59}$ Z. Y. Wei, Z. H. Ni, K. D. Bi, M. H. Chen, and Y. F. Chen, Phys. Lett. A 375, 1195 (2011).

${ }^{60}$ M. Harb, C. Schmising, H. Enquist, A. Jurgilaitis, I. Maximov, P. Shvets, A. Obraztsov, D. Khakhulin, M. Wulff, and J. Larsson, Appl. Phys. Lett. 101, 233108 (2012).

${ }^{61}$ P. K. Schelling, S. R. Phillpot, and P. Keblinski, Phys. Rev. B 65, 144306 (2002)

${ }^{62}$ R. Taylor, Philos. Mag. 13, 157 (1966).

${ }^{63}$ N. Ashcroft and N. Mermin, Solid State Physics (Saunders College Publishing, Fort Worth, 1976).

${ }^{64}$ R. Nicklow, N. Wakabayashi, and H. G. Smith, Phys. Rev. B 5, 4951 (1972).

${ }^{65}$ Eric Pop, Vikas Varshney, and Ajit K. Roy, MRS Bull. 37, 1273 (2012).

${ }^{66}$ CRC Handbook of Chemistry and Physics, 92nd ed., edited by William M. Haynes (CRC, Boca Raton, Florida, 2011).

${ }^{67}$ C. A. Heusch, H. G. Moser, and A. Kholodenko, Nucl. Instrum. Methods Phys. Res., Sect. A 480, 463 (2002).

${ }^{68}$ A. Bosak, M. Krisch, M. Mohr, J. Maultzsch, and C. Thomsen, Phys. Rev. B 75, 153408 (2007).

${ }^{69}$ K. Komatsu, J. Phys. Soc. Jpn. 10, 346 (1955).

${ }^{70}$ S. Huxtable, D. G. Cahill, S. Shenogin, L. Xue, R. Ozisik, P. Barone, M. Usrey, M. S. Strano, G. Siddons, M. Shim, and P. Keblinski, Nat. Mater. 2, 733 (2003)

${ }^{71}$ J. Yang, M. Shen, Y. Yang, W. Evans, W. Chen, A. Zinn, Y. Chen, R. Prasher, T. Xu, P. Keblinski, and D. Li (unpublished).

${ }^{72}$ C. J. Jacobs and G. S. Parks, J. Am. Chem. Soc. 56, 1513 (1934).

${ }^{73}$ P. K. Schelling, S. R. Phillpot, and P. Keblinski, Appl. Phys. Lett. 80, 2484 (2002).

${ }^{74}$ Due to different phonon dispersion for a wave packet of the same frequency, a different wave length is expected in graphene layers; on the other hand, defining wave length in such a confined interface region with only a few atomic layers and two nonnegligible contacts is not very meaningful.

${ }^{75}$ E. Hecht, Optics, 4th ed. (Addison-Wesley, Reading, Massachusetts 2001).

${ }^{76}$ E. T. Swartz and R. O. Pohl, Rev. Mod. Phys. 61, 605 (1989).

${ }^{77}$ Z. Y. Ong and E. Pop, Phys. Rev. B 81, 155408 (2010).

${ }^{78}$ A. Majumdar and P. Reddy, Appl. Phys. Lett. 84, 4768 (2004).

${ }^{79}$ A. J. Schmidt, K. C. Collins, A. J. Minnich, and G. Chen, J. Appl. Phys. 107, 104907 (2010). 
${ }^{80}$ P. E. Hopkins, M. Baraket, E. V. Barnat, T. E. Beechem, S. P. Kearney, J. C. Duda, J. T. Robinson, and S. G. Walton, Nano Lett. 12, 590 (2012).

${ }^{81}$ C. Koitzsch, D. Conrad, K. Scheerschmidt, F. Scharmann, P. Maslarski, and J. Pezoldt, Appl. Surf. Sci. 179, 49 (2001).

${ }^{82}$ H. Zhao and N. R. Aluru, J. Appl. Phys. 105, 104309 (2009).

${ }^{83}$ S. Shin, M. Kaviany, T. Desai, and R. Bonner, Phys. Rev. B 82, 081302(R) (2010).
${ }^{84}$ P. E. Hopkins, T. Beechem, J. C. Duda, K. Hattar, J. F. Ihlefeld, M. A. Rodriguez, and E. S. Piekos, Phys. Rev. B 84, 125408 (2011).

${ }^{85}$ J. C. Duda, C. J. Kimmer, W. A. Soffa, X. W. Zhou, R. E. Jones, and P. E. Hopkins, J. Appl. Phys. 112, 093515 (2012).

${ }^{86}$ M. Lazzeri and F. Mauri, Phys. Rev. B 73, 165419 (2006).

${ }^{87}$ N. Shenogina, P. Keblinski, and S. Garde, J. Chem. Phys. 129, 155105 (2008). 